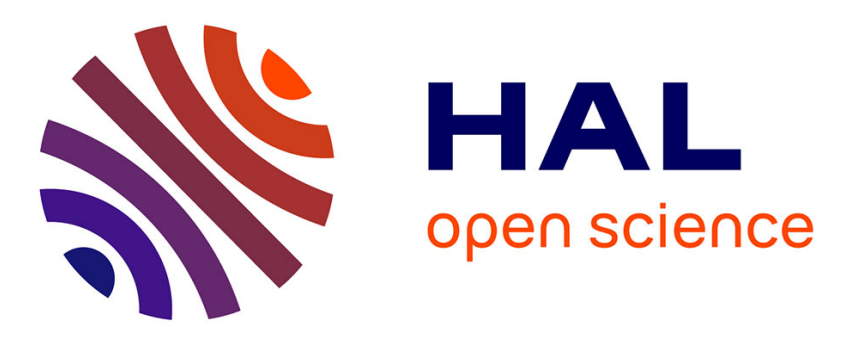

\title{
Model-based approach to describe G-CSF effects in carboplatin-treated cancer patients.
}

Mélanie L. Pastor, Céline M. Laffont, Laurence Gladieff, Antonin Schmitt, Etienne Chatelut, Didier Concordet

\section{- To cite this version:}

Mélanie L. Pastor, Céline M. Laffont, Laurence Gladieff, Antonin Schmitt, Etienne Chatelut, et al.. Model-based approach to describe G-CSF effects in carboplatin-treated cancer patients.. Pharmaceutical Research, 2013, 30 (11), pp.2795-807. 10.1007/s11095-013-1099-z . hal-01137198

\section{HAL Id: hal-01137198 \\ https://hal.science/hal-01137198}

Submitted on 30 Mar 2015

HAL is a multi-disciplinary open access archive for the deposit and dissemination of scientific research documents, whether they are published or not. The documents may come from teaching and research institutions in France or abroad, or from public or private research centers.
L'archive ouverte pluridisciplinaire HAL, est destinée au dépôt et à la diffusion de documents scientifiques de niveau recherche, publiés ou non, émanant des établissements d'enseignement et de recherche français ou étrangers, des laboratoires publics ou privés. 


\title{
Model-Based Approach to Describe G-CSF Effects in Carboplatin-Treated Cancer Patients
}

\author{
Mélanie L. Pastor • Céline M. Laffont • Laurence Gladieff • Antonin Schmitt • Etienne Chatelut • Didier Concordet
}

Received: 20 December 2012 / Accepted: 4 June 2013 / Published online: 26 June 2013

(C) Springer Science+Business Media New York 2013

\begin{abstract}
Purpose Granulocyte colony-stimulating factor (G-CSF) is often used in cancer patients receiving cytotoxic drugs to prevent or reduce high grade neutropenia. We propose a pharmacokinetic/pharmacodynamic model to describe myelotoxicity in both G-CSF treated and non-treated patients that shall increase our understanding of G-CSF effects.

Methods The model was built from absolute neutrophil counts (ANC) obtained in 375 carboplatin-treated patients, 47 of whom received G-CSF. It includes some prior information on G-CSF taken from the literature. Simulations were performed to understand differences in G-CSF effects and explore the impact of G-CSF formulation.

Results Our model well described the data in all patients. Model simulations showed that G-CSF was not as beneficial as expected in some patients. Furthermore, a longer and stronger effect was observed for the pegylated formulation in comparison with the daily standard formulation even if the latter was given for II consecutive days.
\end{abstract}

Electronic supplementary material The online version of this article (doi: | 0. I 007/s | | 095-0 13- | 099-z) contains supplementary material, which is available to authorized users.

M. L. Pastor $(\square)$

Unité de Médecine, Urgences Soins Intensifs

Université de Toulouse, INP, Ecole Nationale Vétérinaire de Toulouse

31076 Toulouse, France

e-mail: m.pastor@envt.fr

M. L. Pastor • C. M. Laffont $\cdot$ D. Concordet

INRA/ENVT, UMRI33I, Toxalim, 31027 Toulouse, France

L. Gladieff • E. Chatelut

Institut Claudius-Regaud, Université de Toulouse, EA4553

20 rue du Pont-Saint-Pierre, 31052 Toulouse, France

A. Schmitt

UFR Sciences Pharmaceutiques et Biologiques, Université de

Bourgogne, 7 Bd Jeanne d'Arc, Dijon 21079, France
Conclusions The proposed model allows a mechanistic interpretation of G-CSF effects on ANC and raises the question of a systematic beneficial effect of G-CSF treatment. Other studies are needed to confirm these findings and help identifying patients for whom G-CSF is beneficial.

KEY WORDS chemotherapy - G-CSF - myelotoxicity . neutropenia · pharmacokinetic/pharmacodynamic (PK/PD) modeling

\section{ABBREVIATIONS}

Abs, Absorption compartment for filgrastim/lenograstim

$\mathrm{Abs}_{2} \quad$ Absorption compartment for pegilgrastim

ANC Absolute neutrophil count

Base Baseline level of absolute neutrophil count

$C_{\text {carbo }} \quad$ Ultrafiltrable circulating (plasma) concentration of carboplatin

$C_{u} \quad$ Free circulating concentration controlling G-CSF effects on bone marrow, calculated as the sum of non-pegylated and pegylated G-CSF free circulating (serum) concentrations

Circ Circulating mature neutrophil count (=ANC)

$E_{\max } 1 \quad$ Maximal effect of non-pegylated or pegylated GCSF on proliferation

$E_{\max } 2 \quad$ Maximal effect of non-pegylated or pegylated GCSF on maturation

$E_{50} 1$ Value of Cu eliciting 50\% of the maximal effect on proliferation

$E C_{50} 2$ Value of Cu eliciting $50 \%$ of the maximal effect on maturation

FI(F2) Absolute bioavailability of filgrastim/lenograstim(pegfilgrastim) after subcutaneous administration (which, in the model, is taking into account via the apparent volume of distribution)

G-CSF Granulocyte colony-stimulating factor 
k Transit rate constant between compartments of granulopoiesis (function of $\mathrm{Cu}$ ) $\left(k=k_{t r} \times\left(1+\frac{E_{\max } 2 \times C_{u}}{E C_{50} 2+C_{u}}\right)\right)$

$k_{a} l(2) \quad$ Absorption rate constant for filgrastim/lenograstim (pegillgrastim)

$k_{\text {circ }} \quad$ Rate constant of elimination of neutrophils from the systemic blood circulation

$K_{D} \quad$ Dissociation constant of RC complex ( $=k_{\text {off }} / k_{\text {on }}$ )

$k_{e l} l \quad$ Rate constant for the linear, non-specific elimination of endogenous G-CSF and filgrastim/lenograstim

$\mathrm{k}_{\mathrm{el}} 2 \quad$ Rate constant for the linear, non-specific elimination of pegfilgrastim

$k_{\text {GCSF }} \quad$ Rate constant of endogenous G-CSF production

$k_{\text {int }} \quad$ Rate constant for non-pegylated or pegylated GCSF elimination after binding to receptors and internalization

$k_{\text {prol }} \quad$ Proliferation rate constant

$k_{\text {tr }} \quad$ "Virtual" transit rate constant when $C_{\mathrm{u}}=0$ (cf. k)

MTT Mean transit time for maturing precursors in bone marrow $\left(M T T=4 / k_{t r} \times\left(1+\frac{E_{\max } 2 \times C_{u}}{E C_{50} 2+C_{u}}\right)\right)$

PK Pharmacokinetic(s)

PKJPD Pharmacokinetic(s)/pharmacodynamic(s)

Prol Stem cell and progenitor cell count (i.e. proliferative cells) in bone marrow

$R \quad$ Concentration in G-CSF receptors present on circulating neutrophils

RC Concentration in bound G-CSF complex (pegylated and non-pegylated G-CSF)

$R_{\max } \quad$ Maximal amount of receptors involved in nonlinear, specific clearance of pegylated and onpegylated G-CSF $(=R+R C)$

RSE Relative standard error

Slope Sensitivity to carboplatin myelotoxicity

Transit $_{\text {I,2,3 }}$ Maturating granulocyte precursor count in transit compartments I, 2 and 3, respectively

$V D_{a} l(2) \quad$ Apparent volume of distribution of G-CSF (pegylated G-CSF) after subcutaneous administration of filgrastim/lenograstim (pegfilgrastim) $\left(V D_{I(2)} / F_{I(2)}\right)$

$\xi \quad$ Proportionality constant for the amount of G-CSF receptors per cell

\section{INTRODUCTION}

Because of the risk of infection, chemotherapy-induced high grade neutropenia is a major concern and requires dose reduction, delays or discontinuation in anticancer drug administration, which may compromise clinical outcome especially in curative regimens (1). In this respect, granulocyte colony-stimulating factor (G-CSF) is often administered to patients receiving cytotoxic drugs in order to prevent or reduce high grade neutropenia which is closely related to febrile neutropenia (2-5).

Exogenous G-CSF reproduces the physiological effect of its endogenous counterpart, that is to say it increases the proliferation of granulocyte progenitors, reduces their maturation time, stimulates the release of precursors from the bone marrow and enhances the survival and function of mature neutrophils, resulting in an increase in absolute neutrophil counts (ANG) $(6,7)$. However, it must be noted that G-CSF only reduces the occurrence of chemotherapy-associated febrile neutropenia $(3-5,8)$ and cannot prevent febrile neutropenia or infections in all patients. Different formulations of G-CSF are available on the market, i.e., daily formulations (e.g. filgrastim, lenograstim) as well as a pegylated once-per-cycle formulation (pegfilgrastim). The current guidelines do not recommend to use one formulation versus another $(8,9)$ but more and more authors suggest that pegfilgrastim would be more effective than the daily forms, most likely due to a better compliance $(4,5,10,11)$. Pegfilgrastim is a sustained-duration form of filgrastim to which a $20 \mathrm{kDa}$ polyethylene glycol molecule is covalently bound to the N-terminal methionine residue. It results that pegfilgrastim has a higher molecular weight $(38.8 \mathrm{kDa})$ than filgrastim (18.8 kDa) and a much higher hydrodynamic radius (12). This leads to a dramatically lower renal clearance of pegfilgrastim compared to filgrastim, with an enhanced serum terminal half-life explaining the sustained duration of effect and the once-per-cycle administration (13). These two proteins share the same binding domain for G-CSF receptor and therefore initiate the same signaling, resulting in the identical mechanism of action.

Pharmacokinetic-pharmacodynamic (PK/PD) modeling has proved very useful to describe the neutrophil timecourse during chemotherapy and predict at-risk neutropenia. Several PK/PD models have been proposed in the past decades (see (14-16) for reviews) but the most popular stays the one of Friberg et al. (17) proposed in 2002. This semimechanistic model uses five compartments to describe the proliferation and maturation of progenitor and precursor cells in the bone marrow as well as the myelotoxicity of anticancer drugs. In this model, the negative feedback mechanism regulating the proliferation of progenitor cells to maintain physiological ANG levels is described using an empirical power function of ANG (i.e. baseline ANC before the start of chemotherapy divided by the current ANG value, the whole raised to a power $\gamma$ ). Actually, this negative feedback is described as corresponding to the effect of endogenous growth hormones, mainly G-CSF. Since G-CSF has also an impact on the maturation of precursors, a recent improvement of Friberg's model has been proposed in the case of docetaxel administration (18). More precisely, the 
power function of ANC ratio has been incorporated not only at the proliferation level but also at the maturation level, allowing an acceleration of maturation rate when $\mathrm{ANC}$ are too low.

Such empirical models are not adapted to describe the effect of exogenous G-CSF and patients receiving exogenous G-CSF are generally excluded from the PK/PD analysis. Because G-CSF is given to patients that are potentially more sensitive to anticancer drugs, the exclusion of such patients may introduce a selection bias, and a model capable to describe neutrophil time-course in case of G-CSF administration is thus highly desirable. Some rough adaptations of Friberg's model have been proposed that incorporate G-CSF treatment as a covariate in the model (19-21). However, the adjunction of G-CSF as a "yes/no" covariate only provides an on/off switch and does not allow the prediction of more subtle adjustments in dose, dosing time and duration of G-CSF treatment. Other, more mechanistic models have been developed for that purpose (15,22-33) but some have been validated in very few cancer patients (26) or in healthy subjects only $(22-24,28,29,31,32)$ or were not very physiological $(15,30)$.

The objective of our work was thus to adapt the widely used Friberg's model to include G-CSF as the driving force for the negative feedback, making the model more physiological and widening its application to every cancer patient irrespective of potential G-CSF treatment during their chemotherapy. Furthermore, such a model shall allow a better understanding of G-CSF effects in cancer patients. Indeed, in our data set, some patients showed unexpectedly low nadir despite G-CSF administration and a relatively high ANC baseline (Fig. 1). Recall that G-CSF has two main effects: it stimulates stem cell proliferation and accelerates maturation time. It is thus possible that an acceleration in maturation time by G-CSF concomitant with a depletion of progenitor cell compartment due to anticancer drug toxicity is not beneficial for the patient: in other terms, G-CSF treatment may not result in a lower and/or shorter neutropenia compared to the absence of G-CSF treatment. The data set used for analysis has been previously analyzed with the Friberg's model after removing the data of patients receiving G-CSF (34). Here, we analyze all data together, accounting for differences in G-CSF formulations (pegylated and daily forms). The model was used to explore the differences between formulations of G-CSF in terms of efficacy.

\section{MATERIALS AND METHODS}

\section{Study Design}

Three hundred and seventy-five cancer patients with various malignant tumors, receiving carboplatin as part of established regimens as monochemotherapy or in combination with different other cytotoxic drugs were recruited in thirteen centers (Supplementary Material 1). Further details are given in (34). Among these patients, 47 were given G-CSF subcutaneously either as a daily formulation (filgrastim (Neupogen $\AA$ ) or

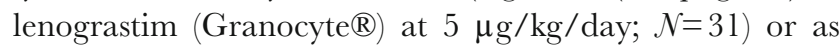
pegfilgrastim (Neulasta ${ }^{\circledR}$ at $6 \mathrm{mg} ; \mathcal{N}=17$ ). G-CSF could be administered on cycle 1 and/or on cycle 2 . All patients who received G-CSF at cycle 2 had experienced a 3/4-grade neutropenia at cycle 1 . The rational for G-CSF administration at cycle 1 was more variable and depended on the clinician's expertise (age, previous chemotherapy, pathological status...). In all 47 patients receiving G-CSF, G-CSF was given at least 1 day after the administration of carboplatin, except for three patients (G-CSF administered the same day). Regarding other cytotoxic drug, G-CSF was given before the end of the etoposide treatment for three additional patients.
Fig. I Observed data for two patients who experienced high grade neutropenia despite G-CSF treatment during their chemotherapy. Carboplatin administrations are indicated by vertical arrows (at day 0 and 54 for patient $\mathbf{a}$, at day 0 and day 35 for patient b). Pegfilgrastim was given subcutaneously to patient $A$ at day 4 (6 mg), and filgrastim was given subcutaneously to patient $\mathbf{b}$ at day 5 during 5 days ( $5 \mu \mathrm{g} / \mathrm{kg} /$ day) and at day 40 during 5 days $(5 \mu \mathrm{g} / \mathrm{kg}$ ) day).
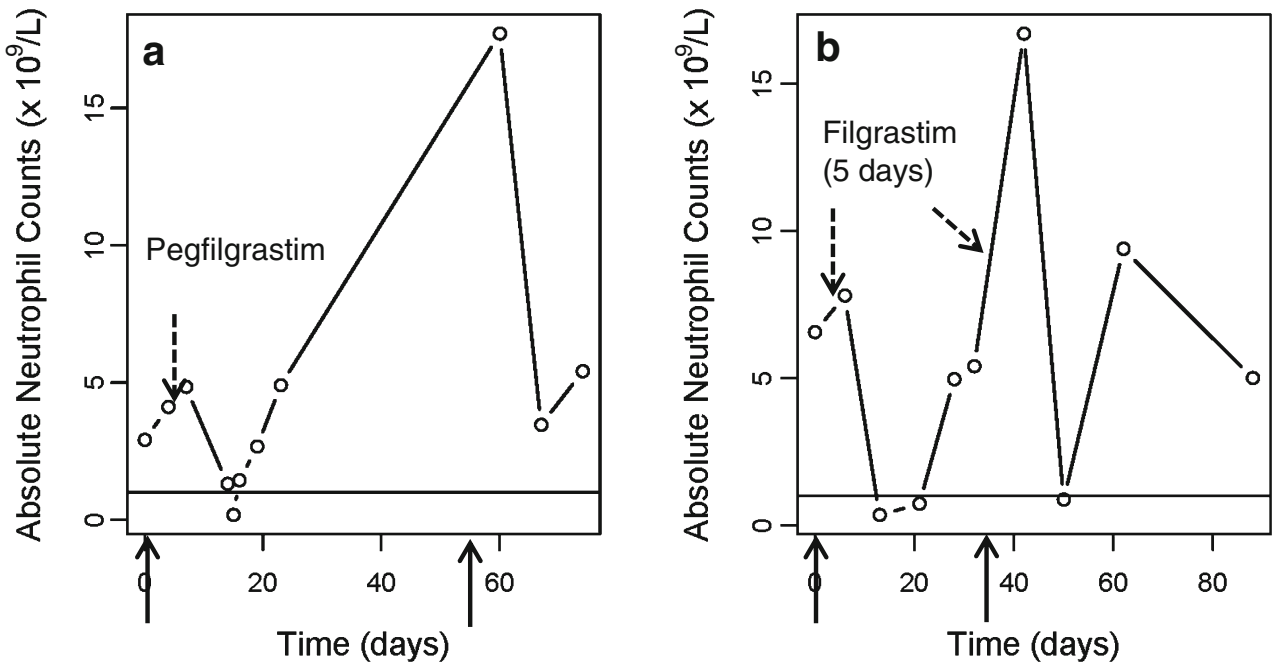
The protocol was approved by the ethical committee of Toulouse and informed written consent was obtained from each patient. The clinical and biological characteristics of individuals receiving or not G-CSF are displayed in Table I and were not significantly different between the two groups.
Carboplatin was given as a single intravenous infusion over 30 or 60 min at the beginning of each cycle with a theoretical intercycle period of 3 weeks. The intravenous doses of carboplatin were adjusted according to the body surface area of the patient or his/her predicted carboplatin clearance, depending on the centers and the regimen. Four blood
Table I Characteristics of the 375 Patients

\begin{tabular}{|c|c|c|c|}
\hline \multirow[t]{2}{*}{ Patients } & \multirow[t]{2}{*}{ Characteristic } & \multicolumn{2}{|c|}{$\begin{array}{l}\text { Mean [Minimum - Maximum] for continuous variables } \\
\text { Number (percentage) for categorical variables }\end{array}$} \\
\hline & & Patients without G-CSF & Patients with G-CSF \\
\hline & Total no. of patients & 328 & 47 \\
\hline & \multicolumn{3}{|l|}{ Sex } \\
\hline & No. Females & $247(75.3 \%)$ & $27(57.4 \%)$ \\
\hline & No. Males & $81(24.7 \%)$ & $20(42.6 \%)$ \\
\hline & Dose of carboplatin (mg) & $547[170-1200]$ & $53 \mid$ [210-955] \\
\hline & Body Surface Area $\left(\mathrm{m}^{2}\right)$ & $1.7[1.3-2.3]$ & $1.7[1.4-2.4]$ \\
\hline & Weight (kg) & $65[40-137]$ & $65[40-123]$ \\
\hline & Height (cm) & $164[\mid 46-187]$ & $165[150-183]$ \\
\hline & Age (years) & $60[21-87]$ & $60[24-83]$ \\
\hline & Neutrophil baseline $\left(\times 10^{9} / \mathrm{L}\right)$ & $5.542[1.357-25.370]$ & $6.069[1.750-13.120]$ \\
\hline & \multicolumn{3}{|l|}{ Primary tumor site } \\
\hline & Ovary & | 46 (44.5\%) & $17(36.2 \%)$ \\
\hline & Uterus & $44(13.4 \%)$ & I (2.0\%) \\
\hline & Lung & $36(11.0 \%)$ & $6(12.8 \%)$ \\
\hline & Soft tissues & $24(7.3 \%)$ & $6(12.8 \%)$ \\
\hline & Others & $62(18.9 \%)$ & $17(36.2 \%)$ \\
\hline & Unknown & $16(4.9 \%)$ & 0 \\
\hline & \multicolumn{3}{|l|}{ Previous chemotherapy } \\
\hline & Yes & I 17 (35.7\%) & $21(44.7 \%)$ \\
\hline & No & $208(63.4 \%)$ & $26(55.3 \%)$ \\
\hline & Unknown & $3(0.9 \%)$ & 0 \\
\hline & \multicolumn{3}{|l|}{ Corticosteroids } \\
\hline & Yes & $64(19.5 \%)$ & I5 (31.9\%) \\
\hline & No & $263(80.2 \%)$ & $32(68.1 \%)$ \\
\hline & Unknown & I (0.3\%) & 0 \\
\hline & \multicolumn{3}{|l|}{ Concomitant chemotherapy } \\
\hline & None & $68(20.7 \%)$ & $4(8.5 \%)$ \\
\hline & Paclitaxel $175 \mathrm{mg} / \mathrm{m}^{2} \mathrm{~b}$ & I 88 (57.3\%) & $13(27.7 \%)$ \\
\hline & Etoposide $120 \mathrm{mg} / \mathrm{m}^{2}$ on days I, 2, $3^{\mathrm{b}}$ & $14(4.3 \%)$ & $14(29.8 \%)$ \\
\hline & Gemcitabine $1,000 \mathrm{mg} / \mathrm{m}^{2}$ on days $1,8^{b}$ & $19(5.8 \%)$ & I (2.1\%) \\
\hline & $\begin{array}{l}\text { Others (fluorouracil, docetaxel, vinorelbine, } \\
\text { trastuzumab, doxorubicin, topotecan, } \\
\text { fotemustine, vinblastine) } \\
\text { Performance status }\end{array}$ & $39(11.9 \%)$ & $15(31.9 \%)$ \\
\hline & 0 & $129(39.4 \%)$ & $21(44.7 \%)$ \\
\hline & । & | 47 (44.8\%) & $16(34.0 \%)$ \\
\hline & 2 & $34(10.4 \%)$ & $5(10.6 \%)$ \\
\hline \multirow{2}{*}{$\begin{array}{l}{ }^{a} \text { Calculated according to the Du- } \\
\text { bois equation }\end{array}$} & 3 & $9(2.7 \%)$ & $3(6.4 \%)$ \\
\hline & Unknown & $9(2.7 \%)$ & $2(4.3 \%)$ \\
\hline
\end{tabular}


samples were taken to measure carboplatin-ultrafiltrable plasma concentrations during the first two cycles: before the start of carboplatin infusion, $5 \mathrm{~min}$ before the end of the infusion and at 1 and $4 \mathrm{~h}$ after the end of the infusion. Cell blood counts were scheduled before chemotherapy and then weekly during the intercycle period.

\section{Population PK/PD Model}

The population PK/PD model was built from the ANC data obtained over the first two cycles in all patients receiving or not G-CSF. Predictions of carboplatin individual PK parameters (POSTHOC values in NONMEM) were obtained from a previous population PK analysis using the model described in (35) for carboplatin-ultrafitrable plasma concentrations.
Our population PK/PD model comprises two parts. The first part describes the proliferation and maturation of progenitor and precursor cells in the bone marrow and was adapted from Friberg's model (17). The other part describes the pharmacokinetics of G-CSF and its effect on bone marrow progenitors and precursors and was adapted from a previous model developed by Krzyzanski et al. (32) for endogenous and exogenous G-CSF. Figure 2 gives a schematic representation of the resulting PK/PD model, including further modifications that will be presented in the Results section. As in Friberg et al. (17), five compartments were used for the first part of the model. One proliferation compartment represents the stem cells and the proliferating precursor cells in the bone marrow. From this compartment, the cells move through three transit compartments, mimicking the maturation in bone marrow, to the blood circulation compartment. Cells are then eliminated

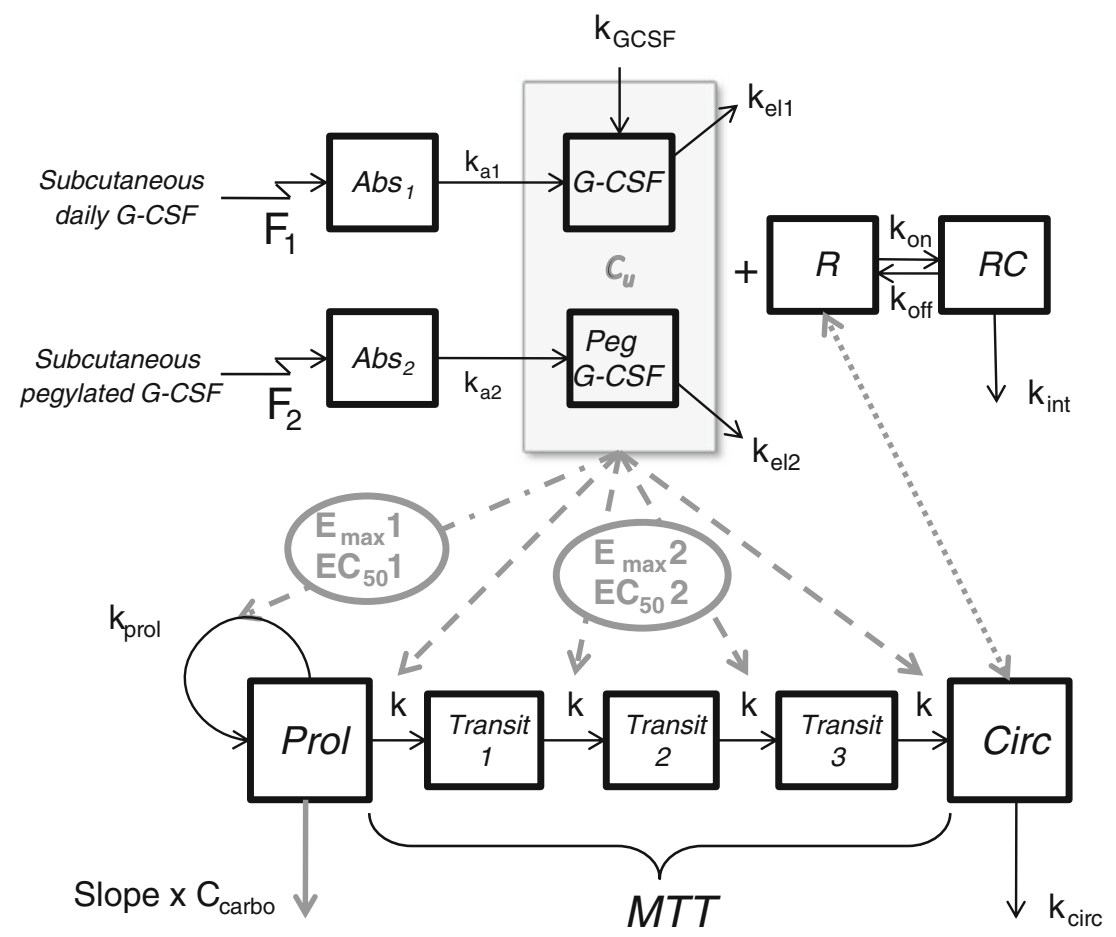

Fig. 2 PK/PD model describing the neutropenic effect of carboplatin with G-CSF-based feedback mechanism. The effect of G-CSF (pegylated or nonpegylated form) on proliferation and maturation processes in bone marrow were assumed to be driven by the free circulating G-CSF concentration. Standard $E_{\max }$ models were used for that purpose (see Supplementary Material 2 for more details). The G-CSF receptor complex RC in blood was assumed to be pharmacologically inactive. Prol: proliferative cells in bone marrow; Transit: maturating granulocyte precursors in bone marrow; Circ: circulating mature neutrophils; MTT: mean transit time for maturing precursors in bone marrow; Slope: sensitivity to carboplatin myelotoxicity; $C_{\text {carbo: }}$ ultrafiltrable plasma concentration of carboplatin; $k_{\text {proll }}$ : proliferation rate constant; $k$ : transit rate constant; $k_{\text {circ }}$ : rate constant of elimination of neutrophils from the systemic circulation; Abs : absorption compartment for filgrastim/lenograstim; Abs2: absorption compartment for pegfilgrastim; FI(F2): bioavailaility of filgrastim/ lenograstim(pegfilgrastim); $C_{u}$ : free circulating concentration controlling G-CSF effects on bone marrow, calculated as the sum of non-pegylated and pegylated G-CSF free circulating (serum) concentrations; R: G-CSF receptors on circulating neutrophils; G-CSF: free circulating G-CSF concentration resulting from endogenous production or from an administration of filgrastim/lenograstim; peg-GCSF: free circulating G-CSF concentration resulting from an administration of pegfilgrastim; RC: bound non-pegylated and pegylated G-CSF assumed here to be pharmacologicaly inactive; $k_{\text {or }} / k_{\text {off: }}$ on/off-rate constants with dissociation constant $K_{D}=k_{o} f\left(k_{o n} ; k_{a} l(2)\right.$ : absorption rate constant for filgrastim/lenograstim (pegfilgrastim); $k_{G C S F}$ : zero-order rate constant of endogenous G-CSF production; $k_{e l} l$ : rate constant for the linear, non-specific elimination of endogenous G-CSF and filgrastim/lenograstim; $k_{e} 2$ : rate constant for the linear, non-specific elimination of pegfilgrastim; $k_{\text {int }}$ : rate constant for non-pegylated or pegylated G-CSF elimination after binding to receptors and internalization; $E_{\max }\left(E_{\max } 2\right)$ : maximal effect of non-pegylated or pegylated G-CSF on progenitor proliferation (precursor maturation); $E C_{50} I\left(E C_{50} 2\right)$ : free non-pegylated or pegylated G-CSF concentration eliciting $50 \%$ of the maximal effect. 
from the systemic blood circulation compartment with a rate constant, $\mathrm{k}_{\text {circ }}$, representing the random movement of cells into the tissue (15). In Friberg's model, the rate constant of transfer between the transit compartments, $\mathrm{k}$, was defined as $(n+1) /$ MTT where $n$ is the number of transit compartments and MTT is the mean transit time or mean maturation time; also, $\mathrm{k}_{\text {circ }}$ was assumed equal to $\mathrm{k}(17)$.

The only difference with Friberg's model lies in the way the regulation of the system by endogenous growth factors, i.e., G-CSF, is modeled. In Friberg's model (15) and its variants (18), the negative feedback on proliferation/maturation rates from the circulating neutrophil counts was described using an empirical function of ANG related to baseline levels. In the present case, we chose a more physiological approach based on circulating G-CSF concentrations. Since no measurements of G-CSF concentrations were available in our study, we used prior information contained in the model developed by Krzyzanski et al. (32) for the disposition of endogenous G$\mathrm{CSF}$ and filgrastim and their effects on bone marrow progenitors and precursors in healthy volunteers. In the model of Krzyzanski et al. (32), the pharmacokinetics of free circulating endogenous G-CSF was described by a one-compartment model with zero-order input $\mathrm{k}_{\mathrm{GCSF}}$ (to mimic the endogenous production of G-CSF) and two mechanisms of elimination: a linear and a non-linear elimination. More precisely, free GCSF could either be eliminated by the kidneys (with first-order rate constant $\mathrm{k}_{\mathrm{el}}$ ) or could bind to receptors $\mathrm{R}$ present on circulating neutrophils and bone marrow precursors (with dissociation constant $\mathrm{K}_{\mathrm{D}}$ ), followed by an internalization and degradation (rate constant $\mathrm{k}_{\mathrm{int}}$ ). In that model, it was assumed that the G-CSF receptor complex, denoted RC, was pharmacologically inactive and that the circulating free G-CSF concentration was the driving force for the effects on stem cells proliferation and maturation. Standard $\mathrm{E}_{\max }$ models were used for these effects, with two different $\mathrm{E}_{\max }$ parameters for proliferation and maturation, noted respectively $E_{\max } 1$ and $E_{\max } 2$, and a single (common) potency parameter $E C_{50}$. In the case of filgrastim administration by the subcutaneous route, an absorption compartment was used with a firstorder absorption rate constant $\mathrm{k}_{\mathrm{a}}$.

In our clinical studies, patients not only received filgrastim but could also receive lenograstim or pegfilgrastim. Identical pharmacokinetic parameters were assumed for filgrastim and lenograstim which are both recombinant forms of endogenous G-CSF and immediate-release daily formulations. Note that filgrastim and lenograstim show the same clinical efficacy at the same dosage (8). Concerning pegfilgrastim, the pegylation results in major differences in the pharmacokinetics with a different absorption rate constant, a different apparent volume of distribution $\left(\mathrm{VD}_{\mathrm{a}}\right)$ and, more importantly, a much reduced renal elimination $(12,29,33)$. To account for these differences in pharmacokinetics, different sets of pharmacokinetic parameters were used for pegfilgrastim and filgrastim/lenograstim with separate absorption and central compartments. In both cases, values of $\mathrm{k}_{\mathrm{a}}, \mathrm{VD}_{\mathrm{a}}$ and $\mathrm{k}_{\mathrm{el}}$ were taken from the literature. Concerning filgrastim/lenograstim, they were denoted $\mathrm{k}_{\mathrm{a}} \mathrm{l}$, $\mathrm{VD}_{\mathrm{a}} 1$ and $\mathrm{k}_{\mathrm{el}} \mathrm{l}$ and fixed to the previously published values in Krzyzanski et al. (32). In the case of pegfilgrastim, they were denoted $\mathrm{k}_{\mathrm{a}} 2, \mathrm{VD}_{\mathrm{a}} 2$ and $\mathrm{k}_{\mathrm{el}} 2$. The values of $\mathrm{k}_{\mathrm{a}} 2$ and $\mathrm{VD}_{\mathrm{a}} 2$ were taken from Roskos et al. (29) while $\mathrm{k}_{\mathrm{el}} 2$ was fixed to $20 \%$ of $\mathrm{k}_{\mathrm{el}} 1$ based on the modeling work of Scholz et al. in man (33).

With respect to the neutrophil-mediated elimination, the same binding and internalization parameters were used for pegylated and non-pegylated G-CSF. Indeed, although the binding to G-CSF receptor is weaker for filgrastim than for pegfilgrastim, this difference is claimed to be marginal (36). We also had to assume similar $\mathrm{EC}_{50}$ and $\mathrm{E}_{\max }$ for pegylated and non-pegylated G-CSF. Thus, in our model, the effects on the bone marrow were mediated by the sum of unbound circulating concentrations of non-pegylated G-CSF (corresponding to filgrastim, lenograstim and endogenously produced G-CSF) and pegylated G-CSF (corresponding to pegfilgrastim).

Different models were tested to describe the cytotoxic effect of carboplatin on proliferative cells, assuming a linear drug effect with a Slope parameter. The observed neutrophil data were log-transformed before the analysis, and an additive error model was used to model residual variability. A lognormal distribution of individual $\mathrm{PK} / \mathrm{PD}$ parameters was assumed. The first-order conditional estimation (FOCE) method in NONMEM 7.2 was used for the estimation of the model parameters. Model development was guided by the objective function value, precision in parameter estimates, graphical assessment and visual predictive checks. The likelihood ratio test was applied to differentiate between two nested models at a significance level $\alpha=0.05$.

Clinical knowledge about the factors that could impact G-CSF prescription (EORTC guidelines $(4,8,37)$ ) was used to preselect covariates: age, sex, corticosteroid multiple ( $>4$ days) administration, monochemotherapy versus polychemotherapy, monochemotherapy versus association with other cytotoxic drugs (paclitaxel, etoposide, gemcitabine, or others), previous chemotherapy, performance status, and comorbidities. The relationship between preselected covariates and the predictions of individual PK/PD parameters were investigated graphically. The covariates for which a relationship was found were then tested in NONMEM using a forward/backward strategy ( $p$-values $<0.05$ and 0.001 , respectively).

\section{Evaluation of the Final PK/PD Model}

Standard goodness-of-fit plots were performed at each step of the model building. The final model was evaluated through visual predictive checks which compare observations with their predictive distribution under the model. Five hundred replicates of the study design were simulated with 
the final model to produce visual predictive checks. Times of ANC measurements were categorized into 15 bins, and medians and 90\% prediction intervals were calculated from the observations and each of the simulated data set within each bin. From the 500 simulated data sets, 95\% confidence intervals were then derived from the computed medians and 90\% prediction intervals within each bin. These 95\% confidence intervals were plotted against time and superimposed on the observations and the percentiles derived from these observations. Finally, normalized prediction distribution errors (NPDE (38)) were computed and their normality assessed through a Quantile-Quantile plot and a histogram.

\section{Simulation-Based Evaluation of the Individual Response to G-CSF}

A series of simulations were performed at the individual level to understand the effect of G-CSF administration upon ANC-time profile compared to the absence of G-CSF treatment. More precisely, the model was applied to patients that received G-CSF in our study to predict what would have been their ANC time profile in the absence of G-CSF treatment (based on their Empirical Bayes Estimates, i.e., the predictions of individual $\mathrm{PK} / \mathrm{PD}$ parameters).

In a second time, the influence of G-CSF formulation was investigated. More specifically, we compared the effect of filgrastim given subcutaneously on day 1 for 11 days (5 $\mu \mathrm{g} / \mathrm{kg} /$ day) with a single administration of pegfilgrastim (6 mg) given subcutaneously on day 1 . All these simulations were performed in a mean patient, that is to say model parameters were set to the population mean estimates. Carboplatin was given on the first day of the first cycle and the dose of carboplatin administered was set to the average dose given to the patients in our study (545 mg). Carboplatin individual PK parameters were set to the average of individual PK parameter predictions.

\section{RESULTS}

\section{Final Population PK/PD Model}

When the model for G-CSF taken from Krzyzanski et al. (32) was implemented as is, our PK/PD model did not provide a very good description of the data. This is the reason why we brought two modifications. The first modification, which led to a considerable improvement in data fitting, was to set the amount of G-CSF receptors involved in the specific elimination of G-CSF $\left(\mathrm{R}_{\max }\right)$ proportional to $\mathrm{ANC}$ and not to the sum of ANG plus the amount of bone marrow precursors as in the original model (32). This modification of the model was supported by previous work showing that ANC drive free G-CSF elimination from the bloodstream and, more generally, drive G-CSF pharmacokinetics during the chemotherapy (39-41). The rational for this new model is discussed in details in the discussion part of the article.

The second modification brought to the model was to estimate distinct $E C_{50} 1$ and $E C_{50} 2$ parameters instead of a single potency parameter. Regarding carboplatin toxicity, carboplatin was assumed to induce a cell loss from the stem cell compartment. The drug effect was proportional to the ultrafiltrable-plasma concentration $\mathrm{C}_{\text {carbo }}$ with the Slope parameter representing the sensitivity to carboplatin myelotoxicity.

To summarize, fixed effect parameters were estimated for Base, $\theta_{M T T_{0}}$, Slope, $E_{\max } 2, E C_{50} 1$ and $E C_{50} 2$. As detailed in Supplementary Material 2, we called $M T T_{0}$ the MTT at baseline, i.e., before any treatment, and $M T T_{0}$ was expressed as $\theta_{M T T_{0}} /\left(1+E_{\max } 2 \times C_{u 0} /\left(E C_{50} 2+C_{u 0}\right)\right)$ where $\mathrm{C}_{\mathrm{u} 0}$ is the free circulating G-CSF concentration at baseline. Note that $E_{\max } 1$ (maximal effect of G-CSF on proliferation) was difficult to estimate from the data and was fixed to the previous estimate from Krzyzanski's (32) model. Other model parameters were fixed to the previously published values as detailed in Table II. The system of differential equations corresponding to our final model with the specification of initial conditions is available in Supplementary Material 2. Moderate interindividual variability was estimated for Base $(30 \%), \theta_{M T T_{0}}(21 \%)$, and Slope $(22 \%)$. The intra-patient variability between cycle 1 and cycle 2, also called inter-occasion variability, could also be estimated for Slope. Only one significant covariate was identified for baseline ANG (Base): multiple corticosteroid administration (yes/no), with an average increase in baseline ANC of $25 \%$ in case of multiple corticosteroid administration. Final model parameter estimates are given in Table II.

The final model was evaluated with standard goodness-of-fit plots (observed vs. individual predicted ANG and observed $v s$. population ANG plots) and visual predictive checks, all displayed in Fig. 3. Standard goodness-of-fit plots look adequate despite a tendency of the model to overestimate low ANC values or, in other terms, underestimate the cytotoxicity of carboplatin. Visual predictive checks show, however, that the model was able to capture the median time-course of the ANC for the two subgroups of patients (with or without G-CSF) even though one median observation was slightly below its $95 \%$ confidence interval for patients off G-CSF around the nadir of the second cycle. Note that model-based confidence intervals were larger for patients on G-CSF due to the lower number of patients in that group $(\mathcal{N}=47)$. Overall, the interindividual variability was well described by the model. Indeed, although some of the 5 th percentiles for observations were above their 95\% confidence interval for patients off G-CSF between 17 and 27 days, the visual predicted checks show the ability of the model to describe and predict the nadir value of neutrophils in both groups of patients, which is of major interest from a clinical point of view. The plots of normalized prediction 
Table II Final Covariate Model

\begin{tabular}{|c|c|c|c|}
\hline & Model Parameters & Value [RSE\%] & IIV [RSE\%] \\
\hline \multirow[t]{7}{*}{ PK-PD estimated parameters } & $\begin{array}{l}\text { Base }=\theta_{1} \times(I-\text { CORT })+\theta_{2} \times \text { CORT } \\
\text { CORT }=0 \text { or I }\end{array}$ & $\begin{array}{l}\theta_{1}=4.88 \times 10^{9} / \mathrm{L}[2.6 \%] \\
\theta_{2}=6.13 \times 10^{9} / \mathrm{L}[4.3 \%]\end{array}$ & $30 \%[12 \%]$ \\
\hline & $\boldsymbol{\theta}_{\mathbf{M T T}_{\mathbf{0}}}=\theta_{3}$ & $\theta_{3}=185 \mathrm{~h}[4.2 \%]$ & $21 \%[20 \%]$ \\
\hline & Slope $=\theta_{5}$ & $\begin{array}{l}\theta_{5}=0.0161[3.9 \%] \mathrm{L} /(\mathrm{mg} . \mathrm{h}) \\
\mathrm{IOV}=34 \%[19 \%]\end{array}$ & $22 \%[56 \%]$ \\
\hline & $\mathbf{E}_{\max } \mathbf{2}=\theta_{6}$ & $\theta_{6}=3.73[17 \%]$ & - \\
\hline & $\mathbf{E C}_{\mathbf{5 0}} \mathbf{I}=\theta_{7}$ & $\theta_{7}=0.0763 \mathrm{ng} / \mathrm{mL}[\mathrm{I} \% \%]$ & - \\
\hline & $\mathbf{E C}_{\mathbf{5 0}} \mathbf{2}=\theta_{8}$ & $\theta_{8}=0.402 \mathrm{ng} / \mathrm{mL}[15 \%]$ & - \\
\hline & Residual variability & $52.1 \%[2.1 \%]$ & - \\
\hline \multirow[t]{10}{*}{ PK parameters of G-CSF (fixed as in (29), (32) and (33)) } & $\mathbf{k}_{\mathrm{a}} \mathbf{l}$ & $0.0228 \mathrm{~h}^{-1}$ & - \\
\hline & $k_{\mathrm{a}} 2$ & $0.651 \mathrm{~h}^{-1}$ & - \\
\hline & $V_{D_{a}} I$ & $5.07 \mathrm{~L}$ & - \\
\hline & $V_{\mathrm{a}_{2}} 2$ & $4.02 \mathrm{~L}$ & - \\
\hline & $\mathbf{k}_{\text {int }}$ & $0.105 h^{-1}$ & - \\
\hline & $\mathbf{k}_{\mathrm{el}} \mathbf{l}$ & $0.152 \mathrm{~h}^{-1}$ & - \\
\hline & $k_{\mathrm{el}} 2$ & $0.2 \times k_{e l} l h^{-1}$ & - \\
\hline & $\mathbf{k}_{\mathrm{D}}$ & I.44 ng/mL & - \\
\hline & $\xi$ & $0.181 \mathrm{fg} / \mathrm{cell}$ & - \\
\hline & $E_{\max } I$ & 34.7 & - \\
\hline
\end{tabular}

RSE relative standard error expressed in \%, IIV interindividual variability, IOV intraindividual (interoccasion) variability, Base baseline level of absolute neutrophil count, CORT I means that the patients received multiple administration of corticosteroids ( $>4$ days) (CORT $=0$ otherwise), $\theta_{\text {MTT }_{0}}$ population parameter for mean transit time at baseline (MTT $)$ such that $M T T_{0}=\theta_{M T T_{0}} / H_{20}$ where $\mathrm{H}_{20}$ is the Hill function for the effect of free G-CSF on maturation rate (see Supplementary Material 2 for more details), Slope sensitivity to carboplatin cytotoxicity, $E_{\text {max }}$ I (2) maximal effect of G-CSF on proliferation (maturation), $E C_{50}$ I (2) free G-CSF concentration eliciting 50\% of the maximal effect on proliferation (maturation), $k_{a} /$ (2) absorption rate constant for the daily (pegylated) formulation, $V D_{a} I(2)$ apparent volume of distribution for the daily (pegylated) formulation, $k_{\text {int }}$ rate constant for non-pegylated and pegylated G-CSF elimination after binding to receptors and internalization, $k_{e l} l$ rate constant for the linear, non-specific elimination of endogenous G-CSF and filgrastim/ lenograstim, $k_{e l} 2$ rate constant for the linear, non-specific elimination of pegfilgrastim, $K_{D}$ dissociation constant of RC complex, $\xi$ proportionality constant for the amount of G-CSF receptors per cell

distribution errors, NPDE, indicated no major departure from the standard normal distribution, showing that the model looked reasonable (plots not shown).

Individual predicted time-profiles of free circulating GCSF concentrations are presented in Fig. 4 for patients off GCSF. Indeed, although there is no interindividual variability for G-CSF PK parameters, the clearance of G-CSF depends on $\mathrm{ANC}$ and that introduces the variability to the individual free G-CSF concentrations. Individual predictions of ANCtime profiles are also displayed in Fig. 4 to highlight how free circulating G-CSF concentrations are inversely correlated with ANC.

\section{Simulation-Based Evaluation of Individual Response to G-CSF}

For the comparison of pegylated and daily formulations, the simulations reveal a longer and stronger effect for pegfilgrastim than for filgrastim even when the latter is given for 11 consecutive days (Fig. 5b and c). Remind that G-CSF increases proliferation rate and transit rate and it is important to assess the difference over time between these rates to understand the difference in neutrophil time-course between the two formulations. As can be seen on Fig. 5f, the difference in profiles between proliferation and transit rates is very small for filgrastim: they reach immediately the same plateau level and, when treatment is stopped, they both decrease very rapidly to baseline levels because of the very high clearance of free G-CSF. On the contrary, when pegfilgrastim is administered, the decrease in free circulating G-CSF concentration is much slower due to a much lower renal clearance resulting from the pegylation. Given the lower $E C_{50}$ for proliferation $\left(E C_{50} 1\right)$ than for maturation $\left(E C_{50} 2\right)$, it follows that the rate of proliferation exceeds the rate of transit time during the terminal phase of pegfilgrastim pharmacokinetics (Fig. 5e). This difference would explain the stronger and faster rebound after pegfilgrastim administration than after filgrastim treatment.

Finally, the simulations predicting what would have been the ANG time-profile without G-CSF for patients receiving G-CSF in our study support the idea that G-CSF was not beneficial in all situations. Three chosen patients are shown 
Fig. 3 Top: Goodness-of-fit plots for observations vs. a) individual predictions and $\mathbf{b}$ ) population predictions of absolute neutrophil counts (ANC). Bottom: Visual predictive checks for ANC $\left(.10^{9} /\right.$ $\mathrm{L})$ for the final model based on 500 simulations of the study design in c) patients off G-CSF and d) patients receiving G-CSF at least once. The circles represent the observed data. The solid lines refer to the median, 5th and 95th percentiles of the observed data. Shaded areas are the 95\% confidence intervals for the median and percentiles 5th and 95th under the model.
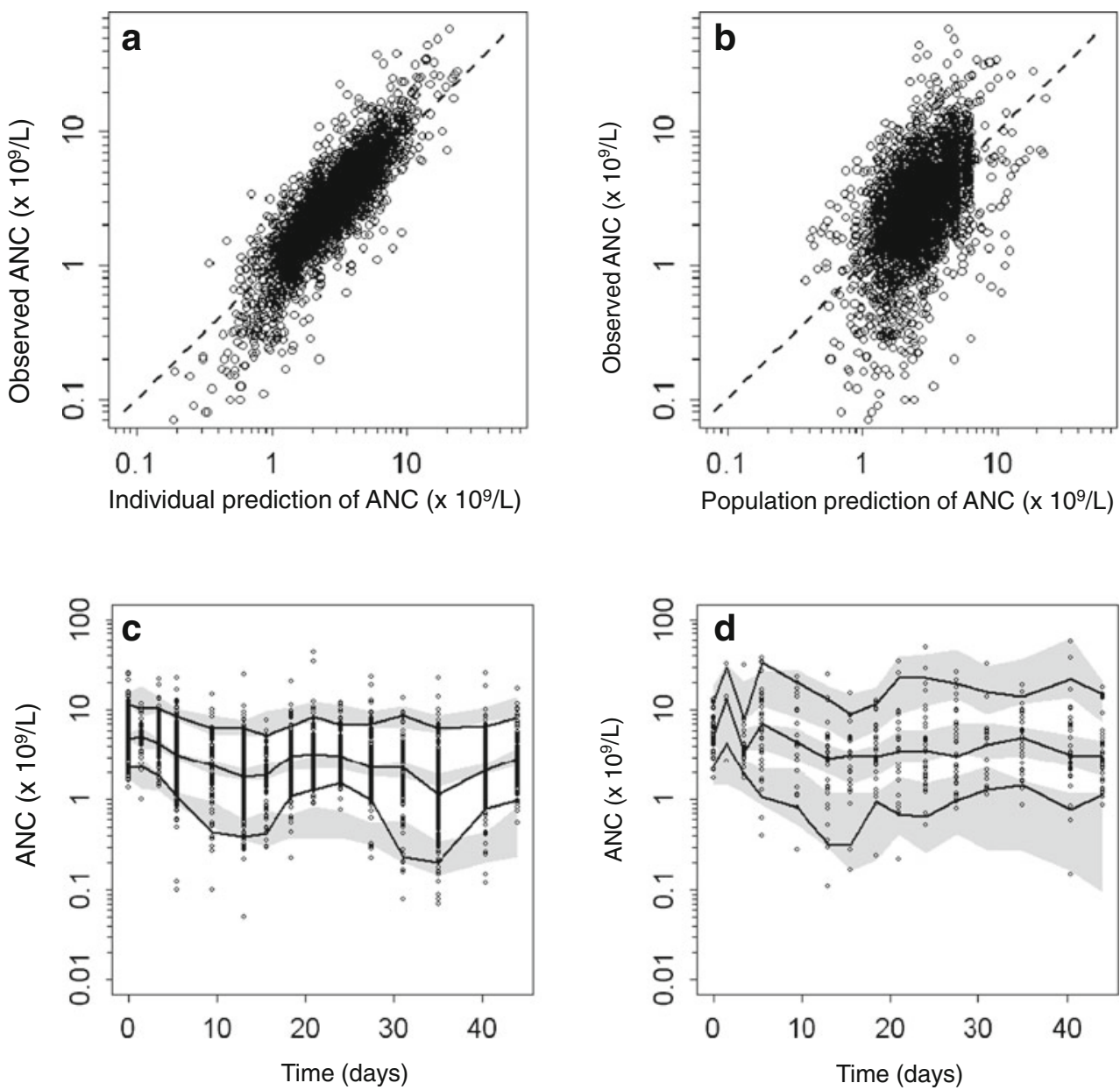

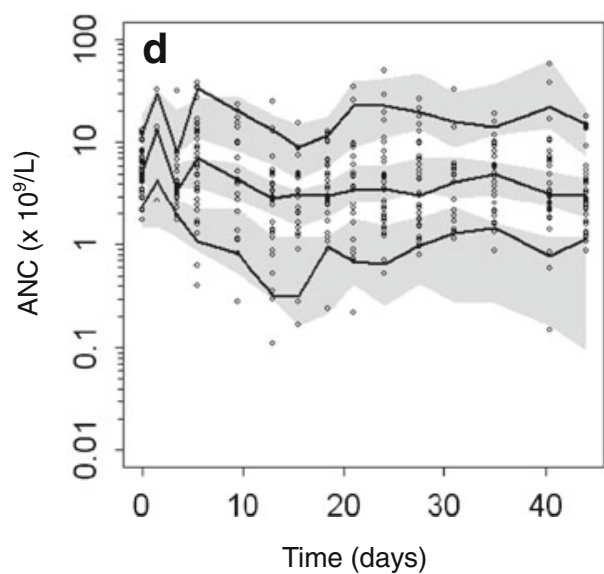

in Fig. 6, illustrating three possible scenarios. For one patient, G-CSF shows some clear benefit, preventing high grade neutropenia at the second cycle. In the second and third patients, G-CSF treatment does not allow any

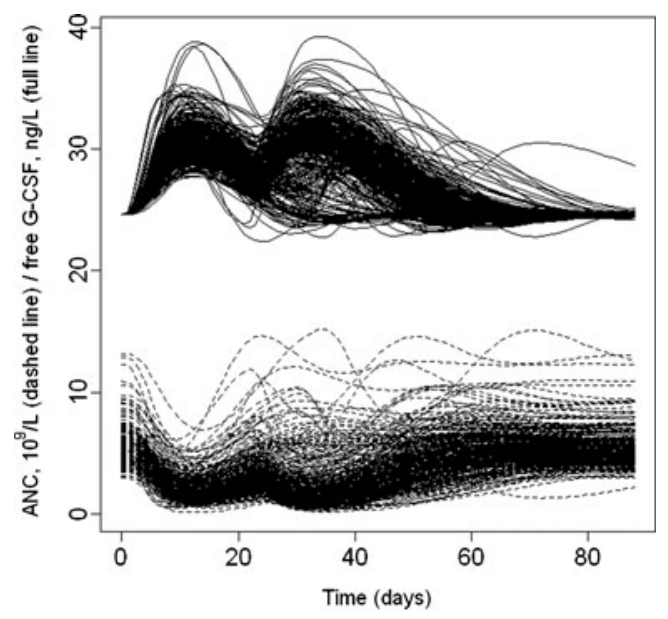

Fig. 4 Individual predicted time-profiles of free circulating G-CSF concentrations and ANC for patients off G-CSF. Note that free circulating G-CSF concentrations are inversely correlated with ANC. improvement in ANC time-profile with respect to low ANC values and the nadir can even be slightly worse.

\section{DISCUSSION}

The population $\mathrm{PK} / \mathrm{PD}$ model presented here was able to correctly describe ANC time course in 375 patients with various tumors treated with carboplatin, whether or not they received G-CSF during their chemotherapy. This model appears more physiological than previously published conventional models (see (14-16) for reviews) because it allows a description of both endogenous and exogenous G-CSF and its effect on the proliferation and maturation of progenitors and precursors in the bone marrow. The power function $(\gamma)$ in Friberg's model (17), representing the negative feedback on the production of mature neutrophils, was replaced by a more physiological representation of endogenous and exogenous G-CSF effects on the proliferation and maturation rates.

The model of Krzyzanski et al. (32) was used as prior information for the disposition and PK/PD of endogenous and exogenous G-CSF. However, a major modification was 

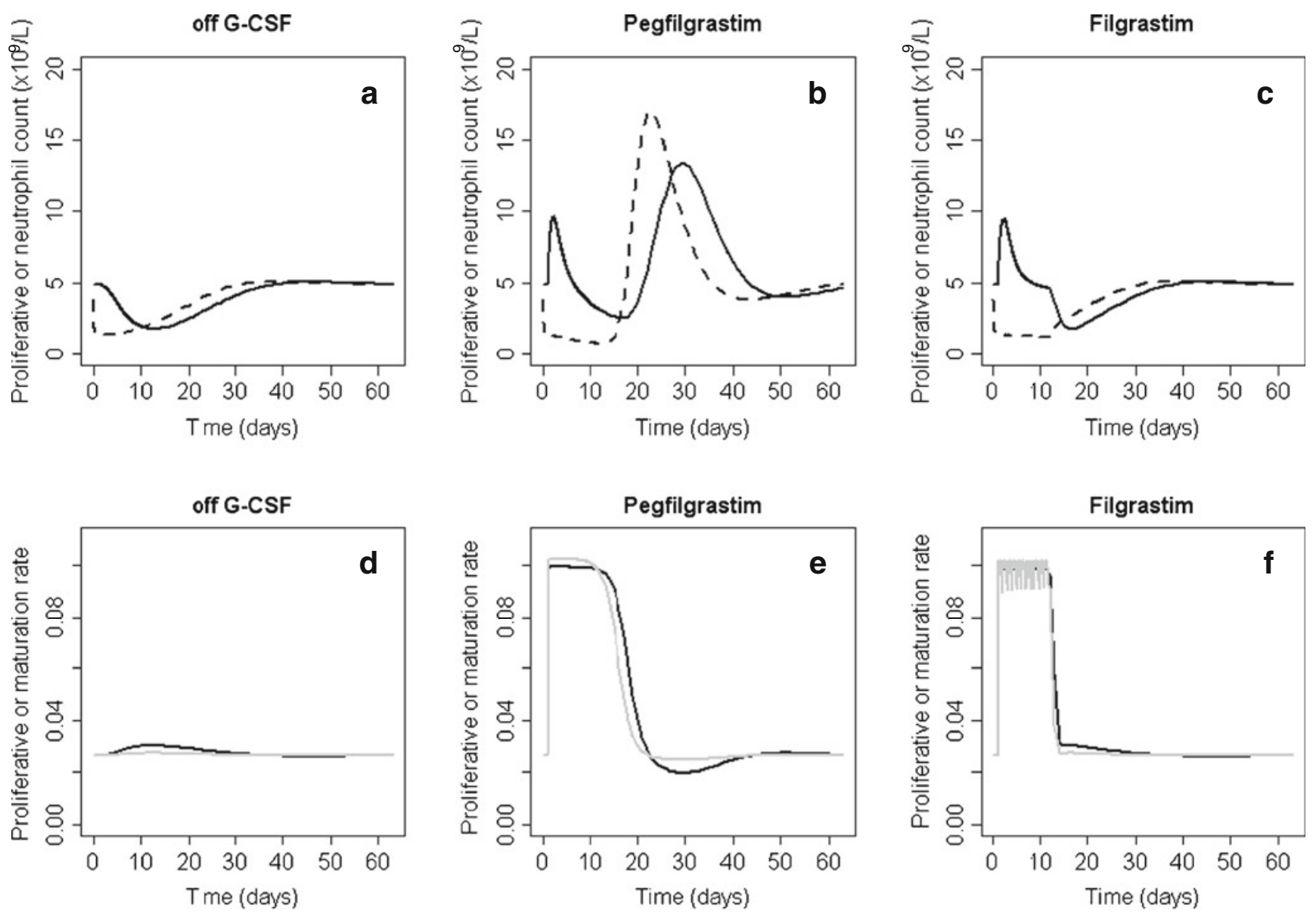

Fig. 5 Effects of subcutaneous pegylated (b, e [6 mg at day I ]) and daily formulations (c, $\mathbf{f}[5 \mu \mathrm{g} / \mathrm{kg} /$ day at day I for I I days $]$ ) compared to the absence of GCSF (a, d) administration in a mean patient. More precisely, we compared the effect of G-CSF formulation (i) on the time-course of proliferative cells (compartment Prol) and circulating neutrophils (compartment Circ) and (ii) on the time-course of proliferation rate (calculated as $\left.k_{\text {prol }} \times\left(1+\frac{E_{\max } 1 \times C_{u}}{E C_{50} 1+C_{u}}\right)\right)$ and maturation rate (calculated as $k_{t r} \times\left(1+\frac{E_{\max } 2 \times C_{u}}{E C_{50} 2+C_{u}}\right)$ and corresponding to $\mathrm{k}$ ). In each case, $545 \mathrm{mg}$ of carboplatin were administered at day 0 . (a, b and $\mathbf{c}$ ) Dashed lines correspond to proliferative cell count in bone marrow (compartment Prol) and the solid lines to the ANC. (d, e and $\mathbf{f}$ ) Black lines correspond to the effect of G-CSF (endogenous and exogenous) on proliferation rate and the grey lines to the effect on maturation (transit) rate.

brought to this model driven by a much better data fitting. This modification was to set the nonlinear component of G-CSG clearance proportional to ANC and not to the sum of ANC and precursor cell counts as in (32). The time course of ANC under chemotherapy was not the same as the time course of precursor cell counts in the bone marrow compartments, and this difference was certainly discriminating in the selection of such a model. The work of Quartino et al. showed indeed mirror patterns for the variations of ANC and endogenous G-CSF serum concentrations over time for cancer patients off G-CSF therapy (18).

Note that the assumption of proportionality between ANC and the nonlinear component of G-CSF clearance has also been made by others $(24,29,31)$. However, the rational for such proportionality is unclear. It has been shown that the amount of G-CSF receptors per cell increases with the maturation and is the highest in circulating neutrophils (42-44). On the other hand, the total pool of G-CSF receptors in the bone marrow needs to be considered. By setting $\mathrm{R}_{\max }$ (pool of receptors involved in G-CSF elimination) proportional to ANC only, the risk is thus to underestimate G-CSF nonlinear clearance. We therefore tried to compensate for this possible underestimation by reestimating $\xi$ (the amount of G-CSF receptors per cell) and obtained a higher value $(5.91 \mathrm{fg} / \mathrm{cell})$ than in Krzyzanski et al. (32), i.e., $0.181 \mathrm{fg} /$ cell. The problem is that the resulting model led to predictions that were not very plausible: especially, it predicted an abnormally high proportion of neutropenia events in patients off G-CSF and almost no prophylactic effect of (peg)filgrastim. These unexpected results shed light on the potential limitations of the structural model of G-CSF itself. In this model, the effect of G-CSF on the bone marrow is driven by the unbound G-CSF concentration $\mathrm{C}_{\mathrm{u}}$. In other terms, what is eliminated by internalization after binding to 

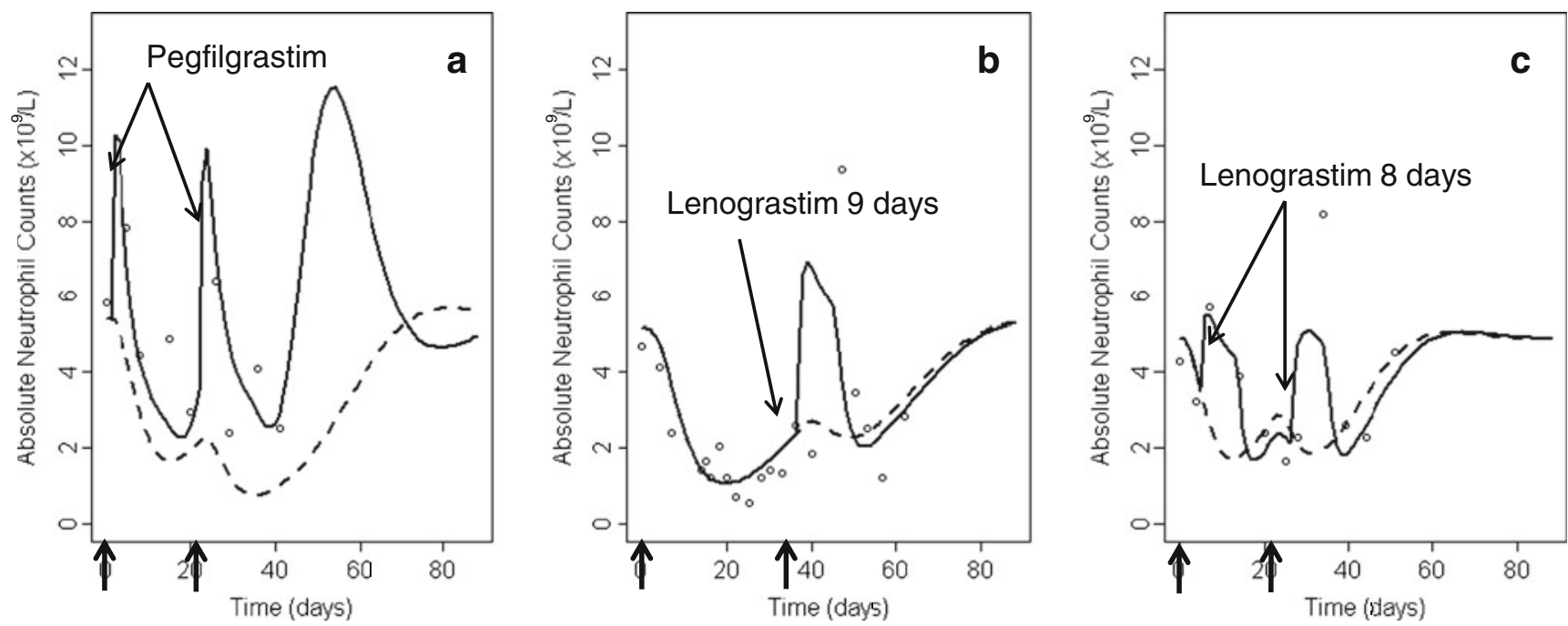

Fig. 6 Prediction of the ANC time-profile without G-CSF for 3 patients who received G-CSF treatment in our data set. The circles correspond to the observations, the solid line to the individual predictions under G-CSF treatment and the dashed line to the individual predictions off G-CSF treatment. Carboplatin administrations are indicated by vertical arrows on the $\mathrm{x}$-axis. (a) Patient who clearly shows benefit from G-CSF treatment. Carboplatin was given at day 0 (583 mg) and day 21 (555 mg), while pegfilgrastim (6 mg) was given subcutaneously at day I and day 22. (b and c) Patients for whom G-CSF treatment does not allow any improvement in the nadir. For patient (b), carboplatin was given at day 0 (660 mg) and day $36(520 \mathrm{mg})$; lenograstim (5 $\mu \mathrm{g} / \mathrm{kg} /$ day) was administered subcutaneously at day 36 for 9 consecutive days. For patient (c), carboplatin was given at day 0 (608 mg) and day 21 (506 mg); lenograstim ( $5 \mu \mathrm{g} / \mathrm{kg} /$ day) was administered subcutaneously at day 5 and day 26 for 8 consecutive days.

G-CSF receptors has no effect. While this is true for blood (receptors on ANC are considered as pharmacologically inactive), this is not true for the bone marrow. A more relevant model would be to model the effect as a function of the fraction bound to bone marrow receptors and not as a function of $\mathrm{C}_{\mathrm{u}}$ as in classical PK/PD models. We did not have the data to build such a model and thus kept the original value of $\xi$ ( $0.181 \mathrm{fg} / \mathrm{cell}$ ) given by Krzyzanski et al. (32). As discussed by the authors themselves, this value (equivalent to 5824 molecules/cell) is higher than the number of receptors per neutrophil found in the literature (30).

Clearly, the main limitation of our work is the lack of GCSF concentration data and the assumption that G-CSF pharmacokinetic parameters were identical in healthy volunteers and cancer patients. It is likely that cancer patients receiving carboplatin may suffer from renal insufficiency since carboplatin is administered in place of cisplatin which cannot be administered to renally impaired patients. Thus, a smaller renal clearance may be encountered in cancer patients treated with carboplatin, resulting in higher G-CSF circulating concentrations and possibly in different nadir values. On the other hand, a sensitivity analysis (not shown) showed the little influence of the first-order rate constant for renal elimination $\left(\mathrm{k}_{\mathrm{el}}\right)$ on nadir values. It is noteworthy that for pegfilgrastim, the renal elimination is very minor, with no change in serum concentration-time profiles among subjects with various degrees of renal impairment (45).

The model of Krzyzanski et al. (32) was originally built for endogenous G-CSF and filgrastim. In the present work, it was extended to describe the effect of pegfilgrastim which was administered to some of the patients in our clinical study. Different sets of pharmacokinetic parameters were used for pegfilgrastim to account for the difference in pharmacokinetics between pegylated and non-pegylated G-CSF. Pegfilgrastim pharmacokinetic parameters were taken from the literature $(29,33)$. Especially, the first-order rate constant for unspecific renal elimination $\left(\mathrm{k}_{\mathrm{el}} 2\right)$ was fixed to $20 \%$ of the one for filgrastim $\left(k_{\mathrm{el}} 1\right)$ based on the modeling work of Scholz et al. in man (33). A difference of the same order of magnitude was observed in the rat: the linear component of pegfilgrastim clearance was $90 \%$ lower than for filgrastim (12).

Despite these limitations, our final model gave reasonable predictions in terms of neutropenia events and (peg)filgrastim effects and well described our clinical data. Efficacy and potency parameters were re-estimated, except for $E_{\max } 1$ (maximal effect on proliferation rate) due to estimability issues. In contrast to the original model for G-CSF (32), two distinct $E C_{50} 1$ and $E C_{50} 2$ parameters were estimated.

Simulation studies were then performed to understand the differences in G-CSF effects and explore the impact of G-CSF formulation. The most surprising finding in these simulation studies was that G-C.SF was not as beneficial as expected in some patients with respect to nadir values. It is well established that G-CSF reduces the maturation time of bone marrow precursors $(6,7)$ and that cytotoxic drugs like carboplatin induce a cell loss from the proliferation compartment (see review (46)). Consequently, it is possible that a 
temporary depletion of stem cell pool occurs after administration of anticancer drug concomitant with or followed by GCSF administration. Such depletion might be problematic in some patients. Additionally, our simulation studies suggest that one administration of pegfilgrastim would be more effective than 11 consecutive administrations of filgrastim. This is consistent with recent publications $(4,5,10,11)$, even if other publications did not evidence any difference between the formulations. A large interindividual variability, leading to low powerful studies, could explain such discrepancies.

Even if our model appears more physiological for the reasons explained above, the increase in model complexity was limited by the available data. Especially, it would have been of interest to include an additional compartment for quiescent cells among progenitor cells (cells in G0 phase). For some patients, this compartment could act as a storage pool of mitotic cells that would limit the depletion of the bone marrow due to the acceleration of the transit time under GC.SF treatment. For other patients, this pool would not be sufficient enough to avoid this depletion. Unfortunately, the available data did not allow such model to be implemented and estimated.

\section{CONCLUSION}

The proposed model allows a mechanistic interpretation of GCSF effects on ANC in carboplatin-treated cancer patients. Especially, it raises the question of a systematic beneficial effect of G-CSF treatment (pegfilgrastim, filgrastim). In other words, some cancer patients do not appear to benefit from GCSF treatment and this could explain why G-CSF does not prevent febrile neutropenia in all patients. Obviously, other studies are needed to confirm these findings and help identifying the patients for whom G-CSF would be beneficial. Finally, our model suggests that pegfilgrastim gives a shorter and weaker neutropenia than the daily formulations, and should probably be preferred. It is clear that our model relies on previous knowledge and assumptions that need to be further documented. However, we believe that such physiological model is the way to go to better understand and predict G-CSF treatment effects in various situations.

\section{REFERENCES}

1. Cameron D. Management of chemotherapy-associated febrile neutropenia. Br J Cancer. 2009;101 suppl 1:S18-22.

2. Segal BH, Freifeld AG, Baden LR, Brown AE, Casper C, Dubberke E, et al. Prevention and treatment of cancer-related infections. J Natl Compr Cancer Netw. 2008;6:122-74.
3. Kelly S, Wheatley D. Prevention of febrile neutropenia: use of granulocyte colony-stimulating factors. Br J Cancer. 2009;101 suppl 1:S6-S10.

4. Aapro M, Crawford J, Kamioner D. Prophylaxis of chemotherapyinduced febrile neutropenia with granulocyte colony-stimulating factors: where are we now? Support Care Cancer. 2010;18:529-41.

5. Cooper KL, Madan J, Whyte S, Stevenson MD, Akehurst RL. Granulocyte colony-stimulating factors for febrile neutropenia prophylaxis following chemotherapy: systematic review and metaanalysis. BMC Cancer. 2011;11:404.

6. Lord BI, Bronchud MH, Owens S, Chang J, Howell A, Souza L, et al. The kinetics of human granulopoiesis following treatment with granulocyte colony-stimulating factor in vivo. Proc Natl Acad Sci U S A. 1989;86:9499-503.

7. Price TH, Chatta GS, Dale DC. Effect of recombinant granulocyte colony-stimulating factor on neutrophil kinetics in normal young and elderly humans. Blood. 1996;88:335-40.

8. Aapro MS, Bohlius J, Cameron DA, Dal Lago L, Donnelly JP, Kearney N, et al. 2010 update of EORTG guidelines for the use of granulocyte-colony stimulating factor to reduce the incidence of chemotherapy-induced febrile neutropenia in adult patients with lymphoproliferative disorders and solid tumours. Eur J Cancer. 2011;47:8-32.

9. Crawford J, Caserta C, Roila F. Hematopoietic growth factors: ESMO recommendations for the applications. Ann Oncol. 2009;20 Suppl 4:162-5.

10. Tan H, Tomic K, Hurley D, Daniel G, Barron R, Malin J. Comparative effectiveness of colony-stimulating factors for febrile neutropenia: a retrospective study. Curr Med Res Opin. 2011;27:79-86.

11. Weycker D, Malin J, Barron R, EdelsbergJ, Kartashov A, Oster G. Comparative effectiveness of filgrastim, pegfilgrastim, and sargramostim as prophylaxis against hospitalization for neutropenic complications in patients with cancer receiving chemotherapy. Am J Clin Oncol. 2012;35:267-74.

12. Yang BB, Lum PK, Hayashi MM, Roskos LK. Polyethylene glycol modification of filgrastim results in decreased renal clearance of the protein in rats. J Pharm Sci. 2004;93:1367-73.

13. Yang BB, Kido A. Pharmacokinetics and pharmacodynamics of pegfilgrastim. Clin Pharmacokinet. 2011;50:295-306.

14. Friberg LE, Karlsson MO. Mechanistic models for myelosuppression. Investig New Drugs. 2003;21:183-94.

15. Shochat E, Rom-Kedar V, Segel LA. G-CSF control of neutrophils dynamics in the blood. Bull Math Biol. 2007;69:2299-338.

16. Testart-Paillet D, Girard P, You B, Freyer G, Pobel G, Tranchand B. Contribution of modelling chemotherapy-induced hematological toxicity for clinical practice. Crit Rev Oncol Hematol. 2007;63:1-11.

17. Friberg LE, Henningsson A, Maas H, Nguyen L, Karlsson MO. Model of chemotherapy-induced myelosuppression with parameter consistency across drugs. J Clin Oncol. 2002;20:4713-21.

18. Quartino AL, Friberg LE, Karlsson MO. A simultaneous analysis of the time-course of leukocytes and neutrophils following docetaxel administration using a semi-mechanistic myelosuppression model. Investig New Drugs. 2012;30:833-45.

19. Sandstrom M, Lindman H, Nygren P, Johansson M, Bergh J, Karlsson MO. Population analysis of the pharmacokinetics and the haematological toxicity of the fluorouracil-epirubicincyclophosphamide regimen in breast cancer patients. Cancer Chemother Pharmacol. 2006;58:143-56.

20. Ramon-Lopez A, Nalda-Molina R, Valenzuela B, Perez-Ruixo JJ. Semi-mechanistic model for neutropenia after high dose of chemotherapy in breast cancer patients. Pharm Res. 2009;26:1952-62.

21. Zandvliet AS, Schellens JH, Copalu W, Beijnen JH, Huitema AD. Covariate-based dose individualization of the cytotoxic drug indisulam to reduce the risk of severe myelosuppression. J Pharmacokinet Pharmacodyn. 2009;36:39-62. 
22. Sugiura M, Yamamoto K, Sawada Y, Iga T. Pharmacokinetic/ pharmacodynamic analysis of neutrophil proliferation induced by recombinant granulocyte colony-stimulating factor (rhG-GSF): comparison between intravenous and subcutaneous administration. Biol Pharm Bull. 1997;20:684-9.

23. Hayashi N, Kinoshita H, Yukawa E, Higuchi S. Pharmacokinetic and pharmacodynamic analysis of subcutaneous recombinant human granulocyte colony stimulating factor (lenograstim) administration. J Clin Pharmacol. 1999;39:583-92.

24. Wang B, Ludden TM, Cheung EN, Schwab GG, Roskos LK. Population pharmacokinetic-pharmacodynamic modeling of filgrastim (r-metHuG-CSF) in healthy volunteers. J Pharmacokinet Pharmacodyn. 2001;28:321-42.

25. Engel C, Scholz M, Loeffler M. A computational model of human granulopoiesis to simulate the hematotoxic effects of multicycle polychemotherapy. Blood. 2004;104:2323-31.

26. Sugiura M, Ohno Y, Yamada Y, Suzuki H, Iga T. Pharmacokinetic/ pharmacodynamic analysis of neutrophil proliferation induced by rhG-CSF in patients receiving antineoplastic drugs. Yakugaku Zasshi. 2004;124:599-604.

27. Scholz M, Engel C, Loeffler M. Modelling human granulopoiesis under poly-chemotherapy with G-CSF support. J Math Biol. 2005;50:397-439.

28. Vainstein V, Ginosar Y, Shoham M, Ranmar DO, Ianovski A, Agur Z. The complex effect of granulocyte colony-stimulating factor on human granulopoiesis analyzed by a new physiologically-based mathematical model. J Theor Biol. 2005;234:311-27.

29. Roskos LK, Lum P, Lockbaum P, Schwab G, Yang BB. Pharmacokinetic/pharmacodynamic modeling of pegfilgrastim in healthy subjects. J Clin Pharmacol. 2006;46:747-57.

30. Shochat E, Rom-Kedar V. Novel strategies for granulocyte colonystimulating factor treatment of severe prolonged neutropenia suggested by mathematical modeling. Clin Cancer Res. 2008;14:6354-63.

31. Foley C, Mackey MC. Mathematical model for G-CSF administration after chemotherapy. J Theor Biol. 2009;257:27-44.

32. Krzyzanski W, Wiczling P, Lowe P, Pigeolet E, Fink M, Berghout A, et al. Population modeling of filgrastim PK-PD in healthy adults following intravenous and subcutaneous administrations. J Clin Pharmacol. 2010;50:101S-12S.

33. Scholz M, Schirm S, Wetzler M, Engel C, Loeffler M. Pharmacokinetic and -dynamic modelling of G-CSF derivatives in humans. Theor Biol Med Model. 2012;9:32.

34. Schmitt A, Gladieff L, Laffont CM, Evrard A, Boyer JC, Lansiaux A, et al. Factors for hematopoietic toxicity of carboplatin: refining the targeting of carboplatin systemic exposure. J Clin Oncol. 2010;28:4568-74.

35. Schmitt A, Gladieff L, Lansiaux A, Bobin-Dubigeon C, EtienneGrimaldi MC, Boisdron-Celle M, et al. A universal formula based on cystatin $\mathrm{C}$ to perform individual dosing of carboplatin in normal weight, underweight, and obese patients. Clin Cancer Res. 2009;15:3633-9.

36. Molineux G. The design and development of pegfilgrastim (PEGrmetHuG-CSF, Neulasta). Curr Pharm Des. 2004;10:1235-44.

37. Aapro MS, Cameron DA, Pettengell R, Bohlius J, Crawford J, Ellis $\mathrm{M}$, et al. EORTC guidelines for the use of granulocyte-colony stimulating factor to reduce the incidence of chemotherapyinduced febrile neutropenia in adult patients with lymphomas and solid tumours. Eur J Cancer. 2006;42:2433-53.

38. Brendel K, Comets E, Laffont C, Laveille C, Mentre F. Metrics for external model evaluation with an application to the population pharmacokinetics of gliclazide. Pharm Res. 2006;23:2036-49.

39. Layton JE, Hockman H, Sheridan WP, Morstyn G. Evidence for a novel in vivo control mechanism of granulopoiesis: mature cell-related control of a regulatory growth factor. Blood. 1989;74:1303-7.

40. Takatani H, Soda H, Fukuda M, Watanabe M, Kinoshita A, Nakamura $\mathrm{T}$, et al. Levels of recombinant human granulocyte colonystimulating factor in serum are inversely correlated with circulating neutrophil counts. Antimicrob Agents Chemother. 1996;40:988-91.

41. Quartino LQ Karlsson MO, Lindman H, and Friberg LE. An integrated G-CSF-myelosuppression model characterizing the target mediated disposition of endogenous G-CSF in breast cancer patients following chemotherapy. PAGE 20 (Athens) abstr 2255, 2011.

42. Avalos BR, Gasson JC, Hedvat C, Quan SG, Baldwin GC, Weisbart RH, et al. Human granulocyte colony-stimulating factor: biologic activities and receptor characterization on hematopoietic cells and small cell lung cancer cell lines. Blood. 1990;75:851-7.

43. Hanazono Y, Hosoi T, Kuwaki T, Matsuki S, Miyazono K, Miyagawa K, et al. Structural analysis of the receptors for granulocyte colony-stimulating factor on neutrophils. Exp Hematol. 1990;18:1097-103.

44. Avalos BR. Molecular analysis of the granulocyte colonystimulating factor receptor. Blood. 1996;88:761-77.

45. Yang BB, Kido A, Salfi M, Swan S, Sullivan JT. Pharmacokinetics and pharmacodynamics of pegfilgrastim in subjects with various degrees of renal function. J Clin Pharmacol. 2008;48:1025-31.

46. Todd RC, Lippard SJ. Inhibition of transcription by platinum antitumor compounds. Metallomics. 2009;1:280-91. 